\title{
Fogitare
}

\section{PREVALÊNCIA DE PRÁTICAS INTEGRATIVAS E COMPLEMENTARES EM PACIENTES SUBMETIDOS À QUIMIOTERAPIA ANTINEOPLÁSICA}

Isabela Oliva Gurgel1', Paola Miranda de Sá2, Paula Elaine Diniz dos Reis ${ }^{3}$, Mariângela Leal Cherchiglia ${ }^{4}$, Ilka Afonso Reis ${ }^{5}$, Ana Lúcia de Mattia ${ }^{6}$, Giovana Paula Rezende Simino ${ }^{7}$

\section{RESUMO}

Objetivo: analisar a prevalência das práticas integrativas e complementares em pacientes que realizam quimioterapia antineoplásica.

Método: estudo quantitativo, observacional, transversal, realizado em um Ambulatório de Quimioterapia de um hospital universitário de Belo Horizonte, Minas Gerais. A amostra foi composta por 70 pacientes com dados obtidos entre outubro de 2017 e maio de 2018. Os dados foram analisados no programa Statistical Package for Social Science utilizando-se estatística descritiva e inferencial.

Resultados: os cânceres predominantes foram mama e cólon e reto. A prevalência de utilização de práticas integrativas foi de $77,1 \%$, sendo a espiritualidade a mais utilizada. O custeio das práticas foi predominantemente não realizado pelo Sistema Único de Saúde. Não foram encontrados fatores associados para utilização de práticas integrativas.

Çonclusão: os pacientes referem benefícios da utilização das práticas integrativas complementares. É relevante que o enfermeiro conheça a prevalência de sua utilização e tenha conhecimento para indicá-las ou contraindicá-las, quando necessário.

DESCRITORES: Terapias complementares; Enfermagem oncológica; Prevalência; Quimioterapia; Oncologia integrativa.

COMO REFERENCIAR ESTE ARTIGO:

Gurgel IO, Sá PM de, Reis PED dos, Cherchiglia ML, Reis IF, Mattia AL de, Simino GPR. Prevalência de práticas integrativas e complementares em pacientes submetidos à quimioterapia antineoplásica. Cogitare enferm. [Internet]. 2019 [acesso em "colocar data de acesso, dia, mês abreviado e ano"]; 24. Disponível em: http:// dx.doi.org/10.5380/ce.v24i0.64450.

Este obra está licenciado com uma Licença Creative Commons Atribuição 4.0 Internacional.

${ }^{1}$ Discente de Enfermagem. Universidade Federal de Minas Gerais. Belo Horizonte, MG, Brasil.

${ }^{2}$ Discente de Enfermagem. Universidade Federal de Minas Gerais. Belo Horizonte, MG, Brasil. 0

${ }^{3}$ Enfermeira. Doutora em Enfermagem. Docente de Enfermagem da Universidade de Brasília. Brasília, DF, Brasil. $\odot$

${ }^{4}$ Médica. Doutora em Saúde Pública. Docente da Universidade Federal de Minas Gerais. Belo Horizonte, MG, Brasil.

${ }^{5}$ Estatística. Doutora em Sensoriamento Remoto. Docente da Universidade Federal de Minas Gerais. Belo Horizonte, MG, Brasil. (-)

${ }^{6}$ Enfermeira. Doutora em Enfermagem. Docente d de Enfermagem da Universidade Federal de Minas Gerais. Belo Horizonte, MG, Brasil. 9

${ }^{7}$ Enfermeira. Doutora em Enfermagem. Docente de Enfermagem da Universidade Federal de Minas Gerais. Belo Horizonte, MG, Brasil. 


\title{
PREVALENCE OF INTEGRATIVE AND COMPLEMENTARY PRACTICES IN PATIENTS UNDERGOING ANTINEOPLASTIC CHEMOTHERAPY
}

\author{
ABSTRACT \\ Objective: To analyze the prevalence of integrative and complementary practices in patients \\ undergoing antineoplastic chemotherapy. \\ Method: A quantitative, observational, cross-sectional study was conducted at a Chemotherapy \\ Outpatient Unit of a university hospital in Belo Horizonte, Minas Gerais. The sample consisted \\ of 70 patients with data obtained between October 2017 and May 2018. Data were analyzed \\ using the SPSS program through descriptive and inferential statistics. \\ Results: The predominant cancers were breast and colon/rectal. The prevalence of integrative \\ practices was $77.1 \%$, with spirituality being the most used. The funding for the practices was \\ predominantly not provided by the Brazilian Nation Health System. No associated factors \\ were found regarding the use of integrative practices. \\ Conclusion: The patients reported benefits of using integrative complementary practices. It \\ is relevant that nurses know the prevalence of their use and have knowledge to indicate or \\ contraindicate them, when necessary.
}

DESCRIPTORS: Complementary therapies; Oncology nursing; Prevalence; Chemotherapy; Integrative oncology.

\section{PREVALENCIA DE PRÁCTICAS DE INTEGRACIÓN Y COMPLEMENTACIÓN EN PACIENTES SOMETIDOS A LA QUIMIOTERAPIA ANTINEOPLASICA}

\begin{abstract}
RESUMEN
Objetivo: analizar la prevalencia de las prácticas de integración y complementación en pacientes de quimioterapia antineoplásica.

Método: estudio cuantitativo, observacional, trasversal, que se realizó en un Ambulatorio de Quimioterapia de un hospital universitario de Belo Horizonte, Minas Gerais. La muestra se compuso por 70 pacientes con datos que se obtuvieron entre octubre de 2017 y mayo de 2018. Se analizaron los datos por medio del programa Statistical Package for Social Science, utilizándose estadística descriptiva y de inferencia.

Resultados: los cánceres predominantes fueron el de mama y colon y recto. La prevalencia de utilización de prácticas integradoras fue de $77,1 \%$, siendo la espiritualidad la más recurrente. El costeo de las prácticas no fue predominantemente realizado por el Sistema Único de Salud. No se encontraron factores asociados para utilización de prácticas integradoras.

Conclusión: los pacientes refieren beneficios de la utilización de las prácticas integradoras complementarias. Es relevante que el enfermero conozca la prevalencia de sus usos y tenga conocimiento para proponerlas o no, cuando necesario.
\end{abstract}

DESCRIPTORES: Terapias complementarias; Enfermería oncológica; Prevalencia; Quimioterapia; Oncología integradora. 
De acordo com a Organização Mundial de Saúde (OMS), as neoplasias malignas constituem hoje a segunda causa de morte no mundo, e está estimado que, em 2018, ela seja responsável por 9,6 milhões de óbitos ${ }^{(1)}$. No Brasil, o câncer é a terceira causa de morte e a segunda causa de morte por doença mais frequente na população. Segundo o Instituto Nacional de Câncer José Alencar Gomes da Silva (INCA), estima-se para o biênio 2018-2019 o surgimento de 600 mil novos casos de câncer para cada ano(2).

Frequentemente o tratamento de escolha para as neoplasias malignas é a quimioterapia antineoplásica. Os quimioterápicos são fármacos com potencial de efeitos tóxicos para células saudáveis e podem ser usados como primeira escolha, em associação com radioterapia ou após a realização de cirurgia. Em 2018, foram registradas 832.183 internações por neoplasias malignas ${ }^{(3)}$.

A OMS recomenda aos países membros que desenvolvam políticas para estimular a medicina tradicional, também chamada de medicina complementar ou medicina integrativa $^{(4)}$. Nos Estados Unidos, em 1998, foi criado o Office of Cancer Complementary and Alternative Medicine (OCCAM) com o objetivo de supervisionar as ações do National Cancer Institute ( $\mathrm{NCl}$ ) para as práticas integrativas e complementares (PIC). O termo Integrative Oncology (IO) foi proposto em 2000 e em 2003, foi fundada a Society for Integrative Oncology (SIO). A SIO compreende um grupo de profissionais, pesquisadores e docentes que realizam práticas e estudos voltados para PIC em oncologia. O MedlinePubmed passa a incorporar o termo Integrative Oncology em 2004(5).

No Brasil, o Sistema Único de Saúde (SUS) implementou, em 2006, a Política Nacional de Práticas Integrativas e Complementares (PNPIC), que possibilitou ampliação da disponibilidade da oferta das PIC aos usuários do SUS ${ }^{(6)}$.

As PIC constituem-se como apoio à terapêutica convencional e incluem uma variedade de conhecimentos e técnicas, que podem ser acessadas de acordo com indicações de profissionais ou pela busca ativa de pessoas saudáveis ou enfermas ${ }^{(4)}$. Na literatura, destacam-se: fitoterapia, acupuntura, homeopatia, arteterapia, meditação, musicoterapia, tratamento naturopático, tratamento osteopático, tratamento quiroprático, reiki, terapia comunitária, yoga, auriculoterapia e massoterapia ${ }^{(6-7)}$.

Vale ressaltar que a prática da medicina moderna ocidental tem possibilitado espaço para o convívio com práticas não convencionais e buscado a intersetorialidade e interdisciplinaridade ${ }^{(8)}$.

Recentemente, dados de 2016 mostram a amplitude dos atendimentos de PIC no SUS, sendo que as mais ofertadas são a medicina tradicional chinesa que inclui a acupuntura (770 mil atendimentos), fitoterapia (85 atendimentos), homeopatia (13 atendimentos). No entanto, 926 mil atendimentos se referiam a outras PIC que não possuíam código próprio para registro, sendo que a portaria n¹45/2017 passa a incluí-los ${ }^{(8-9)}$.

A utilização de PIC nos pacientes com diagnóstico de câncer é elevada e, muitas vezes, acontece sem o conhecimento da equipe multiprofissional de saúde que realiza o tratamento para a neoplasia ${ }^{(10)}$.

Por esse motivo, o objetivo do estudo foi analisar a prevalência de PIC em pacientes que realizam quimioterapia antineoplásica, caracterizando a amostra quanto aos aspectos clínicos e sociodemográficos e verificando a associação destes com a prevalência de PIC. Também propôs-se identificar se há benefícios autorreferidos das PIC para os pacientes que delas faziam uso. 
Trata-se de estudo quantitativo, de caráter observacional, transversal. A coleta de dados foi realizada em um ambulatório de quimioterapia antineoplásica, pertencente ao complexo de um hospital universitário de grande porte de Belo Horizonte-MG, que atende exclusivamente pacientes do SUS.

Os critérios de inclusão dos pacientes foram: idade igual ou superior a 18 anos, ambos os sexos, diagnóstico de neoplasia maligna, realizar protocolos endovenosos no ambulatório de quimioterapia antineoplásica.

Os critérios de exclusão foram: sonolência induzida por medicamento préquimioterápico no momento da entrevista, incapacidade física ou emocional de comunicar-se e punções venosas periféricas que impossibilitassem a assinatura do termo de consentimento livre e esclarecido (TCLE).

O instrumento de coleta de dados utilizado foi construído pelas pesquisadoras e validado, em aparência e conteúdo, por três profissionais especialistas na área de oncologia. $O$ instrumento foi subdividido em dados sociodemográficos, clínicos, relacionados ao tratamento, hábitos de vida e práticas integrativas e complementares. $O$ instrumento continha um questionário aos pacientes, assim como variáveis coletadas dos prontuários. $O$ instrumento foi inserido no programa Epiinfo $₫$ para coleta e armazenamento dos dados por meio de um software android em aparelho móvel. Os dados foram sincronizados e transferidos para o Excel. A amostra de 70 pacientes foi obtida por conveniência no período de outubro de 2017 a maio de 2018.

As variáveis coletadas para identificação sociodemográfica dos pacientes foram: sexo, idade, cor da pele autodeclarada, estado civil, renda mensal per capita (em reais) e fonte de renda. Variáveis de hábitos de vida compreenderam: tabagismo, etilismo. As variáveis clínicas e relacionadas ao tratamento foram: neoplasia maligna, estádio, metástase, cirurgia, radioterapia. As PIC questionadas na pesquisa incluíram: acupuntura, homeopatia, massoterapia, reiki, meditação, tratamento osteopático, arteterapia, musicoterapia, liam gong, quiropraxia, terapia comunitária, yoga, prática espiritual, fitoterapia, auriculoterapia, tai chi chuan e outra modalidade autorreferida pelos pacientes. As variáveis relacionadas a cada PIC incluíram: realização, indicação profissional, tipo de custeio exceto a prática Espiritualidade. Em relação às PIC na visão dos pacientes, as variáveis foram: benefícios e malefícios atribuídos.

Foram realizadas análise descritiva e inferencial. Na análise descritiva, para as variáveis qualitativas foram calculadas frequências absolutas e relativas. Para as variáveis quantitativas, foram calculadas as frequências absolutas e relativas, assim como medidas de dispersão (desvio padrão), medidas de tendência central (média e mediana) e de posição (mínimo e máximo). Na análise bivariada, foi utilizado o Teste Exato de Fisher ou Teste Qui-quadrado. A associação entre variáveis foi considerada estatisticamente significante quando o teste de hipóteses correspondente resultou em valor-p $<0,05$. As análises estatísticas foram realizadas utilizando o programa estatístico Statistical Packpage for Social Science (SPSS) versão 19.0, com auxílio de profissional estatístico.

O estudo atendeu à Resolução n 466/2012 do Conselho Nacional de Saúde, sendo aprovado pelo Comitê de Ética em Pesquisa da Universidade Federal de Minas Gerais, com o número de aprovação do parecer $n^{\circ}$ 2.797.014. A adesão dos pacientes da pesquisa foi voluntária e todos assinaram o TCLE.

\section{RESULTADOS}

Dos 70 pacientes entrevistados, $54(77,1 \%)$ relataram realizar algum tipo de PIC. Os pacientes realizavam predominantemente apenas um tipo de PIC $26(37,1 \%)$. Ainda, 19 $(27,1 \%)$ pacientes da pesquisa realizavam duas PIC, seis pacientes $(8,6 \%)$ três $\mathrm{PIC}$ e três $(4,3 \%)$ pacientes quatro PIC. 
Quanto ao custeio das PIC realizadas, sete (7,4\%) PIC foram custeadas pelo SUS e 40 (42,5\%) foram realizadas por custeio próprio, filantrópico, doação e participação em projeto de pesquisa. Ademais, as PIC do tipo prática espiritual representaram $50 \%$ das PIC, a mais frequente na amostra, sendo que para essa não foi questionado o tipo de custeio. As PIC e sua frequência de utilização estão representadas no Gráfico 1.

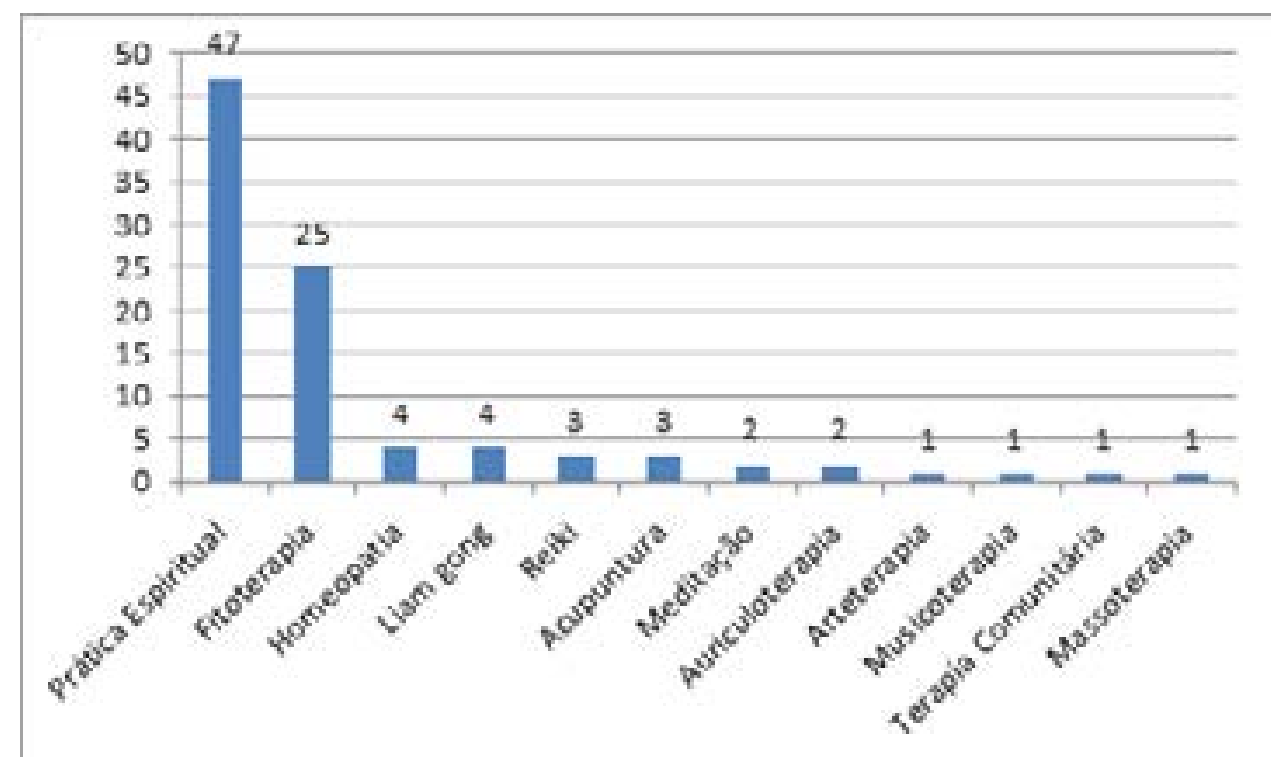

Gráfico 1 - Distribuição dos pacientes do estudo segundo tipo de práticas integrativas e complementares. Belo Horizonte, MG, Brasil, 2018

Com relação à indicação profissional para realização de PIC, as duas práticas mais prevalentes, prática espiritual $(50,0 \%)$ e fitoterapia $(26,6 \%)$, não tiveram indicação profissional. Dentre os pacientes, 10 pacientes relataram que tiveram indicação profissional. As indicações foram para melhora ou controle da dor, sono, alergia, reações da quimioterapia, linfedema e estresse.

A amostra foi composta por 70 pacientes, sendo $41(58,6 \%)$ do sexo feminino. A faixa etária predominante foi de 40 a 59 anos de idade $(48,6 \%)$ e a cor da pele autodeclarada predominantemente foi a parda. A Tabela 1 mostra a frequência e o percentual dos dados sociodemográficos do estudo.

Tabela 1 - Distribuição dos pacientes segundo variáveis sociodemográficas. Belo Horizonte, MG, Brasil, 2018 (continua)

\begin{tabular}{lcc} 
Variável & $\mathbf{n = 7 0}(\mathbf{1 0 0 \% )})$ & $\begin{array}{c}\text { Mediana } \\
\text { (Min; Max) }\end{array}$ \\
\hline Sexo & & \\
\hline Feminino & $41(58,6)$ & ---- \\
\hline Idade & & \\
\hline Média (DP) & $53,8(4,6)$ & $55(20 ; 83)$ \\
\hline Faixa etária & & \\
\hline $20-39$ & $11(15,7)$ & \\
\hline
\end{tabular}




\begin{tabular}{|c|c|c|}
\hline $40-59$ & $34(48,6)$ & \\
\hline$>60$ & $25(35,7)$ & \\
\hline \multicolumn{3}{|l|}{ Cor da pele } \\
\hline Parda & $36(51,4)$ & \\
\hline Branca & $22(31,4)$ & \\
\hline Preta & $10(14,3)$ & \\
\hline Amarela & $2(2,9)$ & \\
\hline \multicolumn{3}{|l|}{ Estado civil } \\
\hline Casado/União estável & $42(60)$ & \\
\hline Solteiro & $19(27,1)$ & \\
\hline Viúvo & $6(8,6)$ & \\
\hline Divorciado/Separado & $3(4,3)$ & \\
\hline \multicolumn{3}{|l|}{ Fonte de renda } \\
\hline Aposentado/pensionista & $27(38,5)$ & \\
\hline Afastamento por doença & $19(27,1)$ & \\
\hline Autônomo & $10(14,3)$ & \\
\hline Não se aplica & $8(11,5)$ & \\
\hline Carteira assinada & $5(7,2)$ & \\
\hline Empregador & $1(1,4)$ & \\
\hline Renda mensal individual & & $\begin{array}{c}954 \\
(0-12000,00)\end{array}$ \\
\hline$\leq 1 \mathrm{SM}$ & $40(57,2)$ & \\
\hline$>1 \mathrm{SM}-\leq 2 \mathrm{SM}$ & $15(21,4)$ & \\
\hline$>2 \mathrm{SM}-\leq 3 \mathrm{SM}$ & $2(2,8)$ & \\
\hline$>3 \mathrm{SM}$ & $7(10)$ & \\
\hline Não sabem/não relataram & $6(8,6)$ & \\
\hline
\end{tabular}

SM= Salário Mínimo; Salário Mínimo vigente em 2018 = R\$ 954,00; DP: desvio-padrão; Min: mínimo. Max: máximo

Quanto aos hábitos de vida, 41 (58,6\%) pacientes relataram que praticaram o uso de bebidas alcoólicas, 21 (30\%) nunca beberam bebidas alcoólicas e oito (11,4\%) ainda utilizavam bebidas alcoólicas concomitante ao tratamento quimioterápico. Dos pacientes que conseguiram parar de utilizar bebidas alcoólicas, $19(27,1 \%)$ mencionaram que o diagnóstico de câncer resultou na interrupção desse hábito, seguido pela força de vontade $15(21,4 \%)$, espiritualidade $(4,3 \%)$ e outros motivos (7,1\%). A utilização anterior de tabaco foi relatada por 31 pacientes do estudo (44,9\%), 34 (48,5\%) nunca fumaram e cinco $(7,1 \%)$ relatam que ainda praticavam a utilização do cigarro concomitante ao tratamento quimioterápico. Dentre os pacientes que conseguiram parar de fumar, $14(20,0 \%)$ atribuíram esta conquista à sua própria força de vontade e $11(15,7 \%)$ ao diagnóstico de câncer, os demais a outros motivos.

Quanto às características clínicas, o câncer mais prevalente foi cólon e reto e mama, com a mesma porcentagem (30\%), o estadiamento IV, a presença de metástase acompanhada de alta incidência de sítios desconhecidos. A cirurgia foi o outro tipo de tratamento mais frequentemente utilizado e a estratégia de tratamento concomitante à quimioterapia. $O$ detalhamento está apresentado na Tabela 2. 
Tabela 2 - Distribuição dos pacientes segundo variáveis clínicas e relacionadas ao tratamento. Belo Horizonte, MG, Brasil, 2018

\begin{tabular}{|c|c|}
\hline Variável & n (\%) \\
\hline \multicolumn{2}{|l|}{ Sítio primário } \\
\hline Cólon e reto & $21(30)$ \\
\hline Mama & $21(30)$ \\
\hline Estômago & $07(10)$ \\
\hline Fígado/vias biliares/pâncreas & $06(8,6)$ \\
\hline Sarcomas & $04(5,7)$ \\
\hline Neoplasias hematológicas & $03(4,3)$ \\
\hline Cabeça e pescoço & $02(2,8)$ \\
\hline Outros tipos & $06(8,6)$ \\
\hline \multicolumn{2}{|l|}{ Estádio } \\
\hline 1 & $02(2,8)$ \\
\hline II & $09(12,9)$ \\
\hline III & $16(22,9)$ \\
\hline IV & $36(51,4)$ \\
\hline Não registrado & $07(10)$ \\
\hline \multicolumn{2}{|l|}{ Metástase } \\
\hline Sim & $39(55,7)$ \\
\hline Não & $31(44,3)$ \\
\hline \multicolumn{2}{|l|}{ Sítio de metástase } \\
\hline Pulmão & $03(7,7)$ \\
\hline Fígado & $03(7,7)$ \\
\hline Osso & $02(5,2)$ \\
\hline Linfonodos & $02(5,2)$ \\
\hline Cerebelo & $01(2,5)$ \\
\hline Região Cervical & $01(2,5)$ \\
\hline Intestino & $01(2,5)$ \\
\hline Peritônio & $01(2,5)$ \\
\hline Região supraclavicular & $01(2,5)$ \\
\hline Múltiplos sítios de metástase & $11(28,2)$ \\
\hline Sítio desconhecidos & $13(33,4)$ \\
\hline \multicolumn{2}{|l|}{ Outros tratamentos } \\
\hline Cirurgia & $56(80)$ \\
\hline Radioterapia & $24(34,3)$ \\
\hline Ambos & $10(14,3)$ \\
\hline \multicolumn{2}{|l|}{ Estratégia de tratamento } \\
\hline Concomitante & $13(54,2)$ \\
\hline Adjuvante & $07(29,2)$ \\
\hline Neoadjuvante & $04(16,6)$ \\
\hline
\end{tabular}


Vale ressaltar que 39 pacientes apresentavam metástase $(55,7 \%)$, o que nos indica que este pode ser o número de pacientes com estádio IV. A verificação da associação entre PIC e variáveis sociodemográficas e clínicas estão apresentadas na Tabela 3.

Tabela 3 - Distribuição dos pacientes segundo associação da utilização de práticas integrativas e complementares realizadas e variáveis sociodemográficas e clínicas. Belo Horizonte, MG, Brasil, 2018

\begin{tabular}{|c|c|c|c|}
\hline Variável & Não n (\%) & $\operatorname{Sim} n(\%)$ & p-valor* \\
\hline Sexo & & & 0,78 \\
\hline Feminino & $10(24,4)$ & $31(75,6)$ & \\
\hline Masculino & $6(20,7)$ & $23(79,3)$ & \\
\hline Estado Civil & & & 0,335 \\
\hline Solteiro & $3(15,8)$ & $16(84,2)$ & \\
\hline Casado/união estável & $10(23,8)$ & $32(76,2)$ & \\
\hline Separado/desquitado/divorciado & 0 & $3(100)$ & \\
\hline Viúvo & $3(50)$ & $3(50)$ & \\
\hline Cirurgia & & & 1 \\
\hline Sim & $13(23,2)$ & $43(76,8)$ & \\
\hline Não & $3(21,4)$ & $11(78,6)$ & \\
\hline Radioterapia & & & 1 \\
\hline Sim & $5(20,8)$ & $19(79,2)$ & \\
\hline Não & $11(24)$ & $35(76)$ & \\
\hline Estádio & & & 0,404 \\
\hline 1 & 0 & $2(100)$ & \\
\hline II & $3(33,3)$ & $6(66,7)$ & \\
\hline III & $5(31,2)$ & $11(68,8)$ & \\
\hline IV & $6(16,6)$ & $30(83,4)$ & \\
\hline Faixa etária & & & 0,554 \\
\hline $20-39$ & $1(9)$ & $10(91)$ & \\
\hline $40-59$ & $8(23,5)$ & $26(76,5)$ & \\
\hline$=$ ou $>60$ & $7(28)$ & $18(72)$ & \\
\hline Renda per capita & & & 0,231 \\
\hline$\leq 1 \mathrm{SM}$ & $9(22,5)$ & $31(77,5)$ & \\
\hline$>1 \mathrm{SM}-\leq 2 \mathrm{SM}$ & $5(33,4)$ & $10(66,6)$ & \\
\hline$>2 \mathrm{SM}-\leq 3 \mathrm{SM}$ & $1(50)$ & $1(50)$ & \\
\hline$>3 \mathrm{SM}$ & 0 & $7(100)$ & \\
\hline
\end{tabular}

Legenda: SM= Salário Mínimo; Salário Mínimo vigente em 2018 = R\$ 954,00

*Teste Qui-Quadrado. 
Vale ressaltar que 51 (94,4\%) dos pacientes que estavam utilizando PIC atribuíram benefícios para sua utilização $(p<0,001)$. Os benefícios atribuídos pelos pacientes foram agrupados em bem-estar geral, tranquilidade, melhora do sono, controle da dor, aumento da fé. Um paciente $(1,85 \%)$ relatou ter sentido malefício com a utilização de PIC, sendo esse um fitoterápico e o malefício foi irritação intestinal. Ainda, dois pacientes não relataram sentir qualquer efeito na utilização da PIC.

\section{DISCUSSÃO}

O perfil da amostra deste estudo representa os pacientes em tratamento com quimioterapia antineoplásica. Os cânceres de mama e próstata são as neoplasias malignas mais incidentes para o sexo feminino e masculino, respectivamente. No entanto, o câncer de próstata possui seu tratamento alicerçado na cirurgia e radioterapia, sendo a quimioterapia antineoplásica recomendada para o cuidado paliativo apenas. Desta maneira, a amostra composta por pacientes com câncer de mama e cólon e reto representa o cenário das neoplasias com maior possibilidade de tratamento quimioterápico no cenário brasileiro ${ }^{(2)}$.

Segundo a Pesquisa Nacional de Saúde de 2013, por sexo, o indicador não apontou diferenças estatísticas entre a proporção percentual de mulheres $(2,0 \%)$ em relação aos homens $(1,6 \%)$ que tiveram diagnóstico de câncer entre 18 anos ou mais de idade no Brasil $^{(11)}$.

Em relação à idade, a população entre 40 e 59 anos teve maior incidência da doença. As taxas ajustadas por idade tanto para homens $(217,27 / 100$ mil) quanto para mulheres (191,78/100 mil) são consideradas intermediárias e compatíveis com as apresentadas para países em desenvolvimento ${ }^{(2)}$. O Registro de Câncer de Base Populacional de Belo Horizonte registrou, para 2012, um aumento crescente de casos de câncer por faixa etária, exceto a partir dos 80 anos quando há um pequeno decréscimo, que se justifica pela própria redução da população(12). A incidência de câncer por faixa etária do presente estudo difere daquela apresentada nas estatísticas, e pode se justificar pelos critérios de exclusão que eliminavam pacientes com impossibilidade de se comunicar física ou emocionalmente.

Com relação à situação conjugal, percebeu-se que a maioria das pessoas referiram ser casadas ou estar em união estável. Estudos mostram que o apoio conjugal e familiar são de extrema importância e possuem papel fundamental na rede de apoio à pessoa com câncer, podendo ajudar nos momentos de fraqueza, fazendo com que a vivência da enfermidade seja amenizada ${ }^{(13)}$.

Quanto à fonte de renda, observamos que a maioria informou ser aposentado ou receber pensão. Também verificamos que a maioria dos pacientes referiram renda mensal individual menor que um salário mínimo. Em relação ao câncer, os segmentos mais pobres enfrentam barreiras de acesso a serviços de saúde para detecção e tratamento precoce da doença $a^{(14)}$.

O alcoolismo e o tabagismo são fatores de risco para diversos tipos de câncer. $O$ diagnóstico de câncer na família leva à busca pela manutenção da saúde. Salienta-se que o tabaco e bebida alcoólica são fatores de risco muito conhecidos para iniciação da carcinogênese, sendo que não há consumo seguro para estas práticas ${ }^{(15)}$.

As estimativas apresentadas para o Brasil refletem perfil semelhante ao de países desenvolvidos, entretanto, ainda convivem com altas taxas de cânceres associados a infecções, que são característicos de países em desenvolvimento. Esse perfil é reflexo das desigualdades regionais tão peculiares ao Brasil, que vão desde diferenças na expectativa de vida, condições socioeconômicas, até acesso aos serviços de saúde para diagnóstico oportuno e tratamento adequado(2).

Quanto à caracterização clínica, os sítios primários predominantes foram mama e cólon e reto, apresentando a mesma porcentagem (30\%), dado que corrobora com a 
epidemiologia do Brasil(2)

A maioria dos pacientes apresentaram estadiamento IV, o que se refere à presença de metástase, com predominância de metástase pulmonar e hepática nos pacientes que apresentavam sítio de metástase conhecido. Tal fato nos indica uma detecção tardia da doença, ou mesmo um início demorado do tratamento, o que remonta novamente aos maiores riscos de adoecimento da população mais pobre, que possui fatores positivos para um maior risco de desenvolvimento de doença, resultante de determinantes sociais de saúde, que contribuem para uma maior dificuldade de acesso ao serviço e uma maior demora em obtenção de diagnóstico e tratamento ${ }^{(14)}$. Segundo o INCA, 60\% do diagnóstico de câncer no Brasil ainda é realizado em estádio avançado da doença, estádio III e IV(2).

A utilização de PIC nos pacientes com diagnóstico de câncer é elevada e, muitas vezes, acontece sem o conhecimento da equipe multiprofissional de saúde que realiza o tratamento para a neoplasia ${ }^{(16-18)}$. Como vimos nesse estudo, as práticas mais prevalentes, prática espiritual e fitoterapia, não tiveram indicação profissional.

Segundo dados do Ministério da Saúde, mais de três mil municípios possuem práticas integrativas e complementares e a distribuição dos serviços está concentrada em 78\% na Atenção Primária. Mais de 8.000 estabelecimentos de saúde ofertam alguma prática integrativa e complementar, o que representa cerca de $19 \%$ das Unidades Básicas de Saúde (UBS) em quase $54 \%$ dos municípios brasileiros, distribuídos pelos 26 estados e Distrito Federal e todas as capitais brasileiras ${ }^{(16)}$.

Dentre as PIC utilizadas, a mais citada foi a Prática Espiritual. O domínio espiritualidade/ religiosidade apresenta-se como item necessário para o melhor enfrentamento, alívio dos impactos provocados pelo câncer e melhor conhecimento para auxiliar os profissionais de saúde. Outrossim, é capaz de oferecer bem-estar e conforto mesmo diante do sofrimento e expectativa da doença(17-18).

Estudo mostrou a relação entre mortalidade e a prática da religiosidade em pacientes com câncer, comparando essa prática com vários comportamentos de saúde. A associação inversa entre frequência de serviços religiosos e mortalidade mostrou-se consistente ao longo do tempo. O comparecimento frequente nos serviços religiosos mostrou associação a um risco significativamente menor de mortalidade por câncer de mama e mortalidade por câncer colorretal(19).

A segunda PIC mais utilizada foi a Fitoterapia. Um estudo do levantamento sobre o uso de plantas medicinais com a terapêutica antineoplásica por pacientes de uma unidade oncológica mostrou que a cultura popular colabora com o uso indiscriminado de plantas medicinais dentro do contexto da automedicação. Nesse mesmo estudo, fez-se observar que os pacientes que fazem uso das plantas durante o tratamento oncológico geralmente $o$ fazem na perspectiva de que possam contribuir para a melhora do seu quadro de saúde ${ }^{(20)}$.

Salienta-se que parte da população dos países em desenvolvimento depende do conhecimento popular para a resolução dos seus problemas de saúde, devido às dificuldades de acesso a médicos e a medicamentos, ou a aspectos culturais da comunidade. A busca de plantas medicinais em farmácias e lojas de produtos naturais é baixa, e o próprio quintal ou o dos amigos representa a principal fonte destes recursos medicinais, provavelmente em razão do baixo custo que representam ${ }^{(21)}$.

Não houve diferença estatisticamente significativa entre características clínicas e sociodemográficas dos pacientes nesse estudo para utilização de PIC. Esse dado também foi encontrado em estudo recente em uma amostra de pacientes oncológicos na Austrália, que ainda destacou o efeito benéfico estatisticamente significativo de PIC autosselecionada pelo paciente medido por meio de instrumentos de qualidade de vida e no sofrimento dos pacientes com câncer ${ }^{(22)}$. Esse resultado possibilita inferir que as PIC são utilizadas pelos pacientes com câncer de uma forma geral, não associado a determinadas características clínicas e sociodemográficas. 
No entanto, outros estudos encontraram associação entre características clínicas e sociodemográficas e utilização de PIC em pacientes com câncer. Em estudo realizado na Alemanha com pacientes com diferentes tipos de neoplasias malignas, foi analisado estatisticamente o uso de PIC associado a sexo, idade, status familiar, educação e renda mensal. A análise revelou uma correlação significativa positiva entre educação (anos de estudo) e utilização de PIC anteriormente ao tratamento e a utilização durante o tratamento para neoplasia(23). Nos Estados Unidos, outro estudo com pacientes em tratamento oncológico verificou associação estatística significativa para utilização de PIC e idade menor ou igual a 65, sexo feminino, escolaridade com nível superior, pessoas com vínculo empregatício, história de cirurgia oncológica e tempo crescente desde o diagnóstico de câncer ${ }^{(24)}$.

Os pacientes referiram que a motivação para realização das PIC estava pautada na diminuição dos efeitos adversos do tratamento oncológico, preservação do sistema imune e da terapêutica tumoral, prevenção do aparecimento de outros problemas de saúde, integração corpo e mente, auxiliar na convivência com a doença, assim como a oportunidade por fazer uma PIC (23,25-26).

Dentre as PIC utilizadas em estudos internacionais recentes em pacientes oncológicos, destaca-se por unanimidade os suplementos alimentares, as vitaminas e a massagem ${ }^{(23,25-26)}$ e a espiritualidade ${ }^{(23)}$, este último corroborando com o presente estudo.

Dentre as limitações do estudo, destaca-se a amostra por conveniência e o desenho do estudo transversal, que não permite acompanhar a utilização de PIC e seus benefícios aos pacientes em tratamento oncológico.

\section{CONCLUSÃO}

Este estudo encontrou alta prevalência de utilização de PIC por pacientes com câncer em tratamento quimioterápico, com destaque para a prática da espiritualidade. Não houve associação entre utilizar PIC e características clínicas e sociodemográficas. Os pacientes referem benefícios à utilização das PIC bem superiores quantitativamente aos malefícios. A indicação profissional das PIC mostrou-se baixa neste estudo.

Desta maneira, estudos que mostrem as PIC realizadas por pacientes com câncer no Brasil são importantes para traçar um panorama de utilização destes serviços que estão predominantemente ofertados na Atenção Primária no SUS. No que concerne à prática de Enfermagem, o conhecimento de evidências relacionadas às PIC e à prevalência de sua utilização por pacientes com câncer em tratamento oncológico pode trazer subsídios para a prática clínica e assistência de enfermagem a esta clientela.

A oncologia integrativa busca a associação do tratamento convencional às PIC, sendo importante ressaltar que devem ser descartadas quando realizarem interferência prejudicial ao tratamento. Torna-se relevante também que o enfermeiro conheça as práticas mais frequentemente utilizadas e tenha conhecimento para indicá-las ou contraindicá-las, quando assim se fizer necessário.

\section{REFERÊNCIAS}

1. Organização Mundial da Saúde (OMS). Câncer. [internet]. 2018. [acesso em 05 nov 2018]. Disponível em: http://www.who.int/en/news-room/fact-sheets/detail/cancer.

2. Ministério da Saúde (BR). Instituto Nacional de Câncer José Alencar Gomes da Silva. Coordenação de Prevenção e Vigilância. Estimativa 2018: incidência de câncer no Brasil. [Internet]. Rio de Janeiro: INCA; 
2017 [acesso em: 05 out 2018]. Disponível em: https://www.inca.gov.br/sites/ufu.sti.inca.local/files// media/document//estimativa-incidencia-de-cancer-no-brasil-2018.pdf.

3. Ministério da Saúde (BR). Sistema de Informações hospitalares do SUS. [Internet]. Brasília: Ministério da Saúde; 2018 [acesso em 22 jun 2019]. Disponível em: tabnet.datasus.gov.br/cgi/tabcgi.exe?sih/cnv/nibr. def.

4. World Health Organization (WHO). WHO Tradicional Medicine Strategy 2014-2023. [Internet]. Geneva: WHO; 2013 [acesso em: 05 out 2018]. Disponível em: http://apps.who.int/iris/ bitstream/10665/92455/1/9789241506090 eng.pdf?ua=1.

5. Wittes R. The National Cancer Institute. National Institutes of Health. Department of Health and Human Services. Integrative Oncology: Cancer Care For The Next Millenium. [Internet]. EUA: The National Cancer Institute; 2000 [acesso em 06 out 2018]. Disponível em: https://www.cancer.gov/about-nci/ legislative/hearings/2000-integrative-oncology.pdf.

6. Ministério da Saúde. Portaria GM/MS n. 971, de 3 de maio de 2006. Política Nacional de Práticas Integrativas e Complementares (PNPIC) no Sistema Único de Saúde. Diário Oficial da União. [Internet]. 03 maio 2006 [ acesso em 06 out 2018]. Disponível em: http://bvsms.saude.gov.br/bvs/saudelegis/gm/2006/ prt0971 0305 2006.html.

7. Ministério da Saúde (BR). Departamento de Atenção Básica. Portaria amplia oferta de PICS. [Internet]. 2017 [acesso em 06 out 2018]. Disponível em: http://dab.saude.gov.br/portaldab/noticias. php? conteudo $=$ \&cod $=2297$.

8. Smith CA, Levett KM, Collins CT, Jones L. Massage, reflexology and other manual methods for pain management in labour. Cochrane Database Syst Rev. [Internet]. 2012 [acesso em 06 out 2018]; 15(2). Disponível em: https://doi.org/10.1002/14651858.CD009290.pub2.

9. Ministério da Saúde (BR). Portaria GM/MS n¹45 de 11 de janeiro de 2017: Altera procedimentos na Tabela de Procedimentos, Medicamentos, Órteses, Próteses e Materiais Especiais do SUS para atendimento na Atenção Básica. Diário Oficial da União, [Internet]. 11 jan 2017 [acesso em 06 out 2018]. Disponível em: http://www.in.gov.br/materia/-/asset publisher/Kujrw0TZC2Mb/content/id/20581305/ do1-2017-01-13-portaria-n-145-de-11-de-janeiro-de-2017-20581242.

10. Leal F, Schwartsmann G, Hiram LS. Medicina complementar e alternativa: uma prática comum entre os pacientes com câncer. Rev Assoc Med Bras. [Internet]. 2008 [acesso em 08 out 2018]; 54(6). Disponível em: http://dx.doi.org/10.1590/S0104-42302008000600007.

11. Ministério do Planejamento. Orçamento e gestão (BR). Instituto Brasileiro de Geografia e Estatística (IBGE). Pesquisa Nacional de Saúde 2013: percepção do estado de saúde, estilos de vida e doenças crônicas. [Internet]. Rio de Janeiro: Instituto Brasileiro de Geografia e Estatística; 2014. [acesso em: 10 ago 2018]. Disponível em: https://biblioteca.ibge.gov.br/visualizacao/livros/liv91110.pdf.

12. Ministério da Saúde (BR). Registro de câncer de base populacional [Internet]. Rio de Janeiro: Instituto Nacional do Câncer Jose Alencar Gomes da Silva [acesso em: 10 ago 2018]. Informações do Registro de Câncer de Base Populacional. Disponível em: http://www2.inca.gov.br/wps/wcm/connect/estatisticas/site/ home/rcbp/.

13. Ferreira NML, Dupas G, Costa DB, Sanchez KOL. Câncer e Família: compreendendo os significados simbólicos. Ciênc. cuid. Saúde. [Internet]. 2010 [acesso em 11 ago 2018]; 9(2). Disponível em: http:// dx.doi.org/10.4025/cienccuidsaude.v9i2.8749.

14. Carvalho CSU. A necessária atenção à família do paciente oncológico. Rev. bras. cancerol. [Internet]. 2008 [acesso em: 10 ago 2018]; 54(1). Disponível em: http://www1.inca.gov.br/rbc/n 54/v01/pdf/ revisao 7 pag 97a102.pdf.

15. Ministério da Saúde (BR). Instituto Nacional de Câncer José Alencar Gomes da Silva (INCA). Câncer de Fígado. [Internet]. Rio de Janeiro: INCA. [acesso em 11 ago 2018]. Disponível em: http://www.inca. gov.br/conteudo view.asp?id=330. 
16. Simino GPR. Práticas integrativas e Complementares em Oncologia. In: Santos M, Corrêa TS, Faria LDBB, Siqueira GSM, Reis PED, Pinheiro RN. Diretrizes Oncológicas 2. ed. São Paulo: Doctor Press Ed. Científica; 2019. p. 819-25.

17. Menezes RR, Kameo SY, Valença TS, Mocó GAA, Santos JMJ. Qualidade de vida relacionada à saúde e espiritualidade em pessoas com câncer. Rev bras cancerol. [Internet]. 2018 [acesso em 12 ago 2018]; 64(1).

18. Mesquita AC, Chaves ECL, Avelino CCV, Nogueira DA, Panzini RG, Carvalho EC. A utilização do enfrentamento religioso/espiritual por pacientes com câncer em tratamento quimioterápico. Rev Latino-Am Enfermagem. [Internet]. 2013 [acesso em 25 jan 2019]; 21(2). Disponível em: http://dx.doi. org/10.1590/S0104-11692013000200010.

19. Li Shanshan, Stampfer MJ, Williams DR, VanderWeele TJ. Religious servisse attendance and mortality among women. JAMA Intern Med. [Internet]. 2016 [acesso em 01 jun 2019]; 176(6). Disponível em: https://dx.doi.org/10.1001\%2Fjamainternmed.2016.1615.

20. Oliveira LAR, Machado RD, Rodrigues AJL. Levantamento sobre o uso de plantas medicinais com a terapêutica anticâncer por pacientes da Unidade Oncológica de Anápolis. Rev bras plantas med. [Internet]. 2014 [acesso em 12 ago 2019]; 16(1). Disponível em: http://dx.doi.org/10.1590/S151605722014000100005.

21. Bieski IGC, Cruz MG. Quintais medicinais: mais saúde, menos hospitais. [Internet] Cuiabá: Governo do Estado de Mato Grosso; 2005 [acesso em 25 jan 2019]. Disponível em: https://www.ppmac.org/sites/ default/files/quintais medicinais.pdf.

22. Stomski NJ, Petterson A, Kristjanson L, Lobb EA, Phillips M, Williams A et al. The effect of selfselected complementary therapies on cancer patients' quality of life and symptom distress: A prospective cohort study in an integrative oncology setting. Complement ther med. [Internet]. 2018 [acesso em 01 jun 2019]; 37. Disponível em: https://doi.org/10.1016/j.ctim.2018.01.006.

23. Kessel KA, Lettner S, Kessel C, Bier H, Biedermann T, Friess $H$, et al. Use of Complementary and Alternative Medicine (CAM) as Part of the Oncological Treatment: Survey about Patients' Attitude towards CAM in a University-Based Oncology Center in Germany. PLoS One. [Internet]. 2016 [ acesso em 20 jun 2019]; 11(11). Disponível em: https://doi.org/10.1371/journal.pone.0165801.

24. Bauml JM, Chokshi S, Schapira MM, Im EO, Li SQ, Langer CJ, et al. Do attitudes and beliefs regarding complementary and alternative medicine impact its use among patients with cancer? A cross-sectional survey. Cancer. [Internet]. 2015 [ acesso em 20 jun 2019]; 121(14). Disponível em: https://doi.org/10.1002/ cncr.29173.

25. Lettner S, Kessel KA, Combs SE. Complementary and alternative medicine in radiation oncology: Survey of patients' attitudes. Strahlenther Onkol. [Internet]. 2017 [acesso em 20 jun 2019]; 193(5).

Disponível em: https://doi.org/10.1007/s00066-017-1101-5.

26. Sun L, Mao JJ, Vertosick E, Seluzicki C, Yang Y. Evaluating Cancer Patients' Expectations and Barriers Toward Traditional Chinese Medicine Utilization in China: A Patient-Support Group-Based Cross-Sectional Survey. Integr Cancer Ther [Internet]. 2018 [acesso em 20 jun 2019]; 17(3). Disponível em: https://doi. org/10.1177/1534735418777117.

Recebido: 04/02/2019

Finalizado: 21/08/2019

Autor Correspondente:

Giovana Paula Rezende Simino

Universidade Federal de Minas Gerais

Av. Alfredo Balena, 190 - 70910900 - Brasília de Minas, MG, Brasil

E-mail: gsimino@yahoo.com.br 
Contribuição dos autores:

Contribuições substanciais para a concepção ou desenho do estudo; ou a aquisição, análise ou interpretação de dados do estudo - IOG, PMS

Elaboração e revisão crítica do conteúdo intelectual do estudo - PEDR, MLC, IAR, ALM

Responsável por todos os aspectos do estudo, assegurando as questões de precisão ou integridade de qualquer parte do estudo - GPRS 